\title{
Steinitz classes of nonabelian extensions of degree $p^{3}$
}

\author{
by
}

\author{
James E. CarTer (Charleston, S.C.)
}

0. Introduction. Let $L / k$ be a finite extension of algebraic number fields. Let $\mathfrak{O}_{L}$ and $\mathfrak{o}$ denote the rings of integers in $L$ and $k$, respectively. As an $\mathfrak{o}$-module, $\mathfrak{O}_{L}$ is completely determined by $[L: k]$ and its Steinitz class $C(L, k)$ in the class group $C(k)$ of $k$ (see [3], Theorem 13). Now let $G$ be a finite group. As $L$ varies over all normal extensions of $k$ with $\operatorname{Gal}(L / k) \simeq G$, $C(L, k)$ varies over a subset $R(k, G)$ of $C(k)$. If we consider only tamely ramified such extensions, then this set is denoted by $R_{\mathrm{t}}(k, G)$. An interesting problem is to determine $R(k, G)$ or $R_{\mathrm{t}}(k, G)$ for various $k$ and $G$. In [7] McCulloh shows that if $G$ is a cyclic group of order $n$, and $k$ contains the multiplicative group $\mu_{n}$ of $n$th roots of unity, then $R(k, G)=R_{\mathrm{t}}(k, G)=$ $C(k)^{d}$ (the subgroup of $C(k)$ consisting of $d$ th powers of elements of $C(k)$ where $d$ is a positive rational integer which depends on $n$ ).

From now on, unless otherwise stated, $p$ will denote an odd prime. In [5] it is shown that when $k$ is any algebraic number field and $G$ is cyclic of order $p$, then $R_{\mathrm{t}}(k, G)$ is again a subgroup of $C(k)$. This result is extended in [6] to include cyclic groups of order $p^{r}$, where $r \geq 1$. In [1] we assume $k$ contains $\mu_{p}$ and $G$ is the nonabelian group of order $p^{3}$ with exponent $p$. There is an exact sequence of groups

$$
\Sigma: 1 \rightarrow A \rightarrow G \rightarrow B \rightarrow 1
$$

where $B$ is cyclic of order $p$. We fix, once and for all, a tamely ramified normal extension $E / k$ with $\operatorname{Gal}(E / k) \simeq B$. As $L$ varies over all tamely ramified normal extensions of $k$ of a particular type which contain $E$, and such that $\operatorname{Gal}(L / k) \simeq G, C(L, k)$ varies over a subset $R_{\mathrm{t}}(E / k, \Sigma)$ of $C(k)$. It is shown that when the ring of integers in $E$ is free as an o-module, then $R_{\mathrm{t}}(E / k, \Sigma)$ is a subgroup of $C(k)$. In the present paper, we continue to assume $k$ contains the appropriate roots of unity, and we return to our consideration of the set $R_{\mathrm{t}}(k, G)$. Making essential use of results of [1] and [2], we will show that $R_{\mathrm{t}}(k, G)$ is always a subgroup of $C(k)$ when $G$ is either of the two nonabelian groups of order $p^{3}$. More specifically, we prove 
the following theorem:

THEOREM 0.1. Let $k$ be an algebraic number field and let $G$ be a nonabelian group of order $p^{3}=m n$ where $n$ is the exponent of $G$. If $\mu_{n} \subseteq k$ then

$$
R_{\mathrm{t}}(k, G)=C(k)^{m(p-1) / 2} .
$$

For the remainder of the paper, the notation will be as introduced above and in [1] and [2].

1. First inclusion. In this section we prove the following proposition:

Proposition 1.1. Let $k$ be any algebraic number field and let $G$ be a nonabelian group of order $p^{3}=m n$ where $n$ is the exponent of $G$. Then

$$
R_{\mathrm{t}}(k, G) \subseteq C(k)^{m(p-1) / 2} .
$$

Pro of. Let $L / k$ be a tamely ramified normal extension with $\operatorname{Gal}(L / k) \simeq$ $G$. Suppose $\mathfrak{p}$ is a prime ideal in $k$ which ramifies in $L / k$, say

$$
\mathfrak{p}=\left(\prod_{i=1}^{g} \mathfrak{P}_{i}\right)^{e}
$$

where the ramification index $e=e\left(\mathfrak{P}_{i}, \mathfrak{p}\right)>1$. Let $f=f\left(\mathfrak{P}_{i}, \mathfrak{p}\right)$ be the residue class degree and let $\mathfrak{D}$ be the different of $L / k$. Since $\mathfrak{p}$ is tamely ramified in $L / k, v_{\mathfrak{P}_{i}}(\mathfrak{D})=e-1$ for each $i$. Therefore

$$
\mathfrak{p}^{f g(e-1)} \| N_{L / k}(\mathfrak{D})=d_{L / k} .
$$

Now suppose $\mathfrak{P}$ is any of the prime ideals in $L$ which divides $\mathfrak{p}$. Since the tame ramification group of $\mathfrak{P}$ over $\mathfrak{p}$ is cyclic of order $e$ it follows that $G$ contains an element of order $e$. Therefore $e \mid n$. Since $m n=p^{3}=e f g$ we have $m \mid f g$. Therefore

$$
C(L, k)=\operatorname{cl}\left(d_{L / k}^{1 / 2}\right) \in C(k)^{m(p-1) / 2} .
$$

2. Second inclusion. Let $k$ and $G$ be as described in the statement of Theorem 0.1. By Proposition 1.1,

$$
R_{\mathrm{t}}(k, G) \subseteq C(k)^{m(p-1) / 2} .
$$

We will now establish the reverse inclusion thereby proving the theorem.

Proposition 2.1. Let $k$ and $G$ be as described in the statement of Theorem 0.1. Then

$$
R_{\mathrm{t}}(k, G) \supseteq C(k)^{m(p-1) / 2} .
$$

Proof. There are two cases to consider.

Case 1 . Suppose $n=p$. Let $\mathfrak{c}$ be any class in $C(k)$. We construct a tamely ramified normal extension $L / k$ such that $\operatorname{Gal}(L / k) \simeq G$ and 
$C(L, k)=\mathfrak{c}^{m(p-1) / 2}$ : by Theorem 2 of $[7]$ there exists a tamely ramified normal extension $E / k$ of degree $p$ such that $C(E, k)=\mathfrak{c}^{(p-1) / 2}$. In Proposition 5 of [1] let $X \in W_{E / k}$ be the trivial class. That proposition gives a tamely ramified normal extension $L / k$ containing $E$ such that $\operatorname{Gal}(L / k) \simeq G$ and $C(L, k)=(\mathfrak{c} X)^{m(p-1) / 2}=\mathfrak{c}^{m(p-1) / 2}$. Therefore

$$
R_{\mathrm{t}}(k, G) \supseteq C(k)^{m(p-1) / 2} .
$$

Case 2. Suppose $n=p^{2}$. In the introduction of [2], the structure of $G$ is described in terms of generators and relations and the parameters $s$ and $l$. According to that description we may assume $s=1$ and $l=1$. Let $\mathfrak{c}$ be any class in $C(k)$. In the following four steps we construct a normal extension $L / k$ as described in Theorem 6 of [2] such that $\operatorname{Gal}(L / k) \simeq G$. We then show in the remaining two steps that $L / k$ is tamely ramified and $C(L, k)=\mathfrak{c}^{m(p-1) / 2}$.

Step 1 . In this step we construct a tamely ramified cyclic extension $E / k$ of degree $p$ such that $C(E, k)=\mathfrak{c}^{(p-1) / 2}$.

Let $\mathfrak{m}=(1-\zeta)^{p^{2}}$. Choose an odd integer $s>3$ such that $\mathfrak{c}^{s}=\mathfrak{c}$. Let $\mathfrak{l}$ be a prime ideal in $\mathfrak{c}$ such that $\mathfrak{l}$ is not a factor of $(p)$. Let $C_{k}(\mathfrak{m})$ be the ray class group modulo $\mathfrak{m}$ of $k$, and let $\mathfrak{c}_{\mathfrak{m}}$ be the element of $C_{k}(\mathfrak{m})$ which contains $\mathfrak{l}$. Choose distinct prime ideals $\mathfrak{l}_{1}, \ldots, \mathfrak{l}_{s}$ in $\mathfrak{c}_{\mathfrak{m}}$. Choose positive integers $u_{i}$, $1 \leq i \leq s$, such that $\left(u_{i}, p\right)=1$ for each $i$ and $\sum_{i=1}^{s} u_{i}=p^{2} s$ (e.g. $u_{i}=p^{2}-1$ for $1 \leq i \leq(s+1) / 2, u_{i}=p^{2}+1$ for $(s+3) / 2 \leq i \leq s-1$, and $\left.u_{s}=p^{2}+2\right)$. Let $\mathfrak{l}_{s+1}$ be a prime ideal in $\mathfrak{c}^{-1}$. Then

$$
(a)=\left(\prod_{i=1}^{s} \mathfrak{l}_{i}^{u_{i}}\right) \mathfrak{l}_{s+1}^{p^{2} s}
$$

where $a \in \mathfrak{o}$ and $a \equiv 1(\bmod \mathfrak{m})$. Let $E=k(\alpha)$ where $\alpha^{p}=a$. Let $\zeta$ be a primitive $p$ th root of unity. By Kummer theory $E / k$ is cyclic of degree $p$ with, say, $\operatorname{Gal}(E / k) \simeq\langle\varrho\rangle$ where $\varrho(\alpha)=\zeta \alpha$. Furthermore, by the proof of Theorem 118 of [4], and by Theorem 119 of [4], the only ramified prime ideals in $E / k$ are the ideals $\mathfrak{l}_{1}, \ldots, \mathfrak{l}_{s}$. Hence, $E / k$ is tamely ramified (in fact, by Theorem 119 of [4], the prime divisors of $(p)$ split completely in $E / k)$. It follows that

$$
d_{E / k}=\left(\prod_{i=1}^{s} \mathfrak{l}_{i}\right)^{p-1}
$$

Therefore, as in the proof of Lemma 4 of [1], we have

$$
C(E, k)=\operatorname{cl}\left(d_{E / k}^{1 / 2}\right)=\operatorname{cl}\left(\prod_{i=1}^{s} \mathfrak{l}_{i}\right)^{(p-1) / 2}=\mathfrak{c}^{s(p-1) / 2}=\mathfrak{c}^{(p-1) / 2} .
$$

Step 2. In this step we construct the element $\kappa$. Let $\mathfrak{q}$ be a prime ideal in $\mathfrak{c}^{-1}$ such that $\mathfrak{q}$ is not a factor of $(p)$. Note that $\left(\mathfrak{c}^{-1}\right)^{s}=\left(\mathfrak{c}^{s}\right)^{-1}=\mathfrak{c}^{-1}$ 
where $s$ is the integer of Step 1 . Let $\mathfrak{c}_{\mathfrak{m}}^{\prime}$ be the class in $C_{k}(\mathfrak{m})$ which contains $\mathfrak{q}$ and choose distinct prime ideals $\mathfrak{q}_{1}, \ldots, \mathfrak{q}_{s}$ in $\mathfrak{c}_{\mathfrak{m}}^{\prime}$ such that $\left(\mathfrak{q}_{i}, \mathfrak{l}_{j}\right)=1$ for $1 \leq i \leq s$ and $1 \leq j \leq s+1$ where the $\mathfrak{l}_{j}$ are the prime ideals of Step 1. Choose positive integers $v_{i}$ for $1 \leq i \leq s$ such that $\left(v_{i}, p\right)=1$ and $\sum_{i=1}^{s} v_{i}=p s$. Let $\mathfrak{q}_{s+1}$ be a prime ideal in $\left(\mathfrak{c}_{\mathfrak{m}}^{\prime}\right)^{-1}$ such that $\left(\mathfrak{q}_{s+1}, \mathfrak{l}_{j}\right)=1$ for $1 \leq j \leq s+1$. We have

$$
(\kappa)=\left(\prod_{i=1}^{s} \mathfrak{q}_{i}^{v_{i}}\right) \mathfrak{q}_{s+1}^{p s}
$$

where $\kappa \in \mathfrak{o}$ and $\kappa \equiv 1(\bmod \mathfrak{m})$. Since $\left((\kappa), d_{E / k}\right)=1$ each $\mathfrak{q}_{i}$ remains prime or splits completely in $E / k$.

Step 3. In this step we construct the element $e$. In the proof of Proposition 5 of [1], let $X \in W_{E / k}$ be the trivial class, $\mathfrak{b}=(\alpha \kappa)$, and $\mathfrak{m}=(1-\zeta)^{p^{2}}$. Construct $e$ as outlined in that proof. Then

$$
(e)=\left(\prod_{i=1}^{t} \mathfrak{P}_{i}^{b_{i}}\right) \mathfrak{Q}^{p t}
$$

as described there.

Step 4. It is straightforward to verify that with the elements constructed in the above three steps, the conditions of Theorem 6 of [2] are satisfied (see, for instance, the paragraph preceding Example 1 of [2]). Consequently, we obtain a normal extension $L / k$ as described in that theorem with $\operatorname{Gal}(L / k) \simeq G$.

Step 5. In this step we show that no prime divisor of $(p)$ ramifies in the extension $L / k$. Hence, $L / k$ is tamely ramified. In fact, we will show that we can arrange for all prime divisors of $(p)$ to split completely in $L / k$.

Assume

$$
(1-\zeta)=\prod_{i=1}^{g} \mathfrak{p}_{i}^{w_{i}}
$$

where the $\mathfrak{p}_{i}$ are distinct prime ideals in $k$ and the $w_{i}$ are positive integers. Let $\mathfrak{p}=\mathfrak{p}_{1}$ and $w=w_{1}$. Thus

$$
v_{\mathfrak{p}}(1-\zeta)=w .
$$

Recall from Step 1 that the prime divisors of $(p)$ split completely in $E / k$. Hence $\mathfrak{p} \mathfrak{O}_{E}=\mathfrak{P}^{N}$ where $\mathfrak{P}$ is a prime ideal in $E$. Since $a \equiv 1(\bmod \mathfrak{m})$, (2.5) implies that $a \equiv 1\left(\bmod \mathfrak{p}^{w p^{2}}\right)$. Hence

$$
\mathfrak{p}^{w p+x} \mid(a-1)=\left(\alpha^{p}-1\right)=\prod_{k=0}^{p-1}\left(\alpha-\zeta^{k}\right)
$$


where $x=w p(p-1)$. It follows that

$$
\mathfrak{P}^{w p+x} \mid \prod_{k=0}^{p-1}\left(\alpha-\zeta^{k}\right)
$$

and therefore

$$
\mathfrak{P}^{w+1} \mid\left(\alpha-\zeta^{i}\right)
$$

for some $i$. For $i \neq j$ we have $\left(\alpha-\zeta^{i}\right)-\left(\alpha-\zeta^{j}\right)=\zeta^{j}\left(1-\zeta^{i-j}\right)$. Therefore, by $(2.5)$,

$$
\mathfrak{P}^{w} \|\left(\alpha-\zeta^{i}\right)-\left(\alpha-\zeta^{j}\right) .
$$

Thus, by (2.7) and (2.8), $\mathfrak{P}^{w} \|\left(\alpha-\zeta^{j}\right)$ whenever $j \neq i$. Therefore,

$$
v_{\mathfrak{P}}\left(\prod_{j \neq i}\left(\alpha-\zeta^{j}\right)\right)=(p-1) w .
$$

By (2.6) we have

$$
v_{\mathfrak{P}}\left(\prod_{k=0}^{p-1}\left(\alpha-\zeta^{k}\right)\right) \geq w p+x
$$

Hence

$$
\begin{aligned}
v_{\mathfrak{P}}\left(\prod_{k=0}^{p-1}\left(\alpha-\zeta^{k}\right)\right) & =v_{\mathfrak{P}}\left(\prod_{j \neq i}\left(\alpha-\zeta^{j}\right)\right)+v_{\mathfrak{P}}\left(\alpha-\zeta^{i}\right) \\
& =(p-1) w+v_{\mathfrak{P}}\left(\alpha-\zeta^{i}\right) \geq w p+x .
\end{aligned}
$$

It follows that $v_{\mathfrak{P}}\left(\alpha-\zeta^{i}\right) \geq w+x$. Therefore

$$
\mathfrak{P}^{w+x} \mid\left(\alpha-\zeta^{i}\right) \text {. }
$$

Hence, $\alpha \equiv \zeta^{i}\left(\bmod \mathfrak{P}^{w+x}\right)$. Since $\kappa \equiv 1(\bmod \mathfrak{m}), e^{-N} \equiv 1(\bmod \mathfrak{m})$, and $e^{\theta} \equiv 1(\bmod \mathfrak{m}),(2.4)$ implies that $\kappa \equiv 1\left(\bmod \mathfrak{P}^{w p^{2}}\right), e^{-N} \equiv 1$ $\left(\bmod \mathfrak{P}^{w p^{2}}\right)$, and $e^{\theta} \equiv 1\left(\bmod \mathfrak{P}^{w p^{2}}\right)$. Since $w p^{2} \geq w p+1$ and $w+x=$ $w+w p(p-1) \geq w p+1$, we obtain $c \equiv \zeta^{i}\left(\bmod \mathfrak{P}^{w p+1}\right)$ and $b \equiv \zeta$ $\left(\bmod \mathfrak{P}^{w p+1}\right)$. Since $\zeta \equiv 1\left(\bmod E^{p}\right), \mathfrak{P}$ splits completely in $M / E$ and $K / E$ by Theorem 119 of [4]. By the Galois theory of prime decomposition in algebraic number fields, it follows that $\mathfrak{p}$ splits completely in $L / k$. Therefore, every prime divisor of $(p)$ splits completely in $L / k$. In particular, no prime divisor of $(p)$ ramifies in $L / k$. Therefore $L / k$ is tamely ramified.

Step 6. We now show that $C(L, k)=\mathfrak{c}^{m(p-1) / 2}$. From Step 1 the prime factors $\mathfrak{l}_{i}$ of $(a), 1 \leq i \leq s$, are distinct and are contained in the class $\mathfrak{c}$ of $C(k)$. Furthermore, each $\mathfrak{l}_{i}$ totally ramifies in $E / k$. Let $\mathfrak{l}_{i} \mathfrak{O}_{E}=\mathfrak{L}_{i}^{p}$ where $\mathfrak{L}_{i}$ is a prime ideal in $E$. From Step 2 the prime factors $\mathfrak{q}_{i}$ of $(\kappa), 1 \leq i \leq s$, are distinct and are contained in the class $\mathfrak{c}^{-1}$ of $C(k)$. Furthermore, each $\mathfrak{q}_{i}$ either remains prime or splits completely in $E / k$. Assume $\mathfrak{q}_{i}$ remains prime 
in $E / k$ for $1 \leq i \leq r \leq s$, say, $\mathfrak{q}_{i} \mathfrak{O}_{E}=\mathfrak{Q}_{i}$, and $\mathfrak{q}_{j}$ splits completely in $E / k$ for $r+1 \leq j \leq s$, say, $\mathfrak{q}_{j} \mathfrak{O}_{E}=\mathfrak{Q}_{j}^{N}$, where $\mathfrak{Q}_{j}$ is some prime ideal in $E$. From Step 3 the prime factors $\mathfrak{P}_{i}$ of $(e)$ are distinct and split completely in $E / k$, say, $\mathfrak{p}_{i} \mathfrak{O}_{E}=\mathfrak{P}_{i}^{N}$. Moreover, $\mathfrak{p}_{i}$ is a prime ideal in $k$ which is contained in the trivial class $X \in W_{E / k}$, and such that $i \neq j$ implies $\mathfrak{p}_{i} \neq \mathfrak{p}_{j}$. Finally, by construction, the ideals $(a),(\kappa)$, and $(e)$ are pairwise relatively prime, and they are each prime to $(p)$. We can now describe $d_{L / E}$. We have $K=E(\beta)$ where $\beta^{p}=b=\zeta e^{-N}$. Since

$$
(b)=\left(\prod_{i=1}^{t} \mathfrak{P}_{i}^{-b_{i} N}\right) \mathfrak{Q}^{p t N}
$$

where $\left(b_{i}, p\right)=1$ for each $1 \leq i \leq t$, it follows by the proof of Theorem 118 of [4] that the prime ideals in $E$ which ramify in $K / E$ are precisely the prime factors of the ideals $\mathfrak{P}_{i}^{N}$ for $1 \leq i \leq t$. Therefore, by the first part of the proof of Proposition 3 of [1],

$$
\left(\prod_{i=1}^{t} \mathfrak{P}_{i}^{p(p-1) N}\right) \| d_{L / E} .
$$

Furthermore, the only other possible prime factors of $d_{L / E}$ are prime ideals in $E$ which ramify in $M / E$. By Theorem 118 of [4] these will be among the prime factors of $(c)$ where $c=\kappa \alpha e^{\theta}$. Since the prime factors of $\left(e^{\theta}\right)$ are included in the set of prime factors of $(b)=\left(e^{-N}\right)$, which all ramify in $L / E$, their contribution to $d_{L / E}$ is given by (2.9). It remains to determine the contribution made to $d_{L / E}$ from $(\kappa)$ and $(\alpha)$. Arguing as in the case of the extension $K / E$, we obtain

$$
\left(\prod_{i=1}^{s} \mathfrak{L}_{i}^{p(p-1)}\right) \| d_{L / E}
$$

and

$$
\left(\prod_{i=1}^{r} \mathfrak{Q}_{i}^{p(p-1)}\right)\left(\prod_{i=r+1}^{s} \mathfrak{Q}_{i}^{p(p-1) N}\right) \| d_{L / E} .
$$

Taking the product of the factors appearing in (2.9)-(2.11) we obtain $d_{L / E}$. Since $N_{E / k}\left(\mathfrak{L}_{i}\right)=\mathfrak{l}_{i}, N_{E / k}\left(\mathfrak{Q}_{i}\right)=\mathfrak{q}_{i}^{p}$ for $1 \leq i \leq r, N_{E / k}\left(\mathfrak{Q}_{i}^{N}\right)=\mathfrak{q}_{i}^{p}$ for $r+1 \leq i \leq s$, and $N_{E / k}\left(\mathfrak{P}_{i}^{N}\right)=\mathfrak{p}_{i}^{p}$, we have, letting $\delta=(p-1) / 2$,

$$
\begin{aligned}
C(L, k) & =C(E, k)^{[L: E]} \mathfrak{N}_{E / k}(C(L, E))=\mathfrak{c}^{p^{2} \delta} \mathfrak{N}_{E / k}\left(\operatorname{cl}\left(d_{L / E}^{1 / 2}\right)\right) \\
& =\mathfrak{c}^{p^{2} \delta} \operatorname{cl}\left(N_{E / k}\left(d_{L / E}^{1 / 2}\right)\right)=\mathfrak{c}^{p^{2} \delta} \mathfrak{c}^{s p \delta} \mathfrak{c}^{-s p^{2} \delta} X^{t p^{2} \delta} \\
& =\mathfrak{c}^{p^{2} \delta} \mathfrak{c}^{p \delta} \mathfrak{c}^{-p^{2} \delta} X^{p^{2} \delta}=\mathfrak{c}^{p \delta}=\mathfrak{c}^{m(p-1) / 2} .
\end{aligned}
$$

Hence, $R_{\mathrm{t}}(k, G) \supseteq C(k)^{m(p-1) / 2}$. 
Acknowledgements. This work appears as part of the author's Ph.D. thesis. He would like to thank Professors Leon R. McCulloh and Stephen V. Ullom for their comments and suggestions.

\section{References}

[1] J. E. Carter, Steinitz classes of a nonabelian extension of degree $p^{3}$, Colloq. Math. 71 (1996), 297-303.

[2] - Characterization of Galois extensions of prime cubed degree, Bull. Austral. Math. Soc. 55 (1997), 99-112.

[3] A. Fröhlich and M. J. Taylor, Algebraic Number Theory, Cambridge University Press, 1991.

[4] E. Hecke, Lectures on the Theory of Algebraic Numbers, Springer, 1981.

[5] R. L. Long, Steinitz classes of cyclic extensions of prime degree, J. Reine Angew. Math. 250 (1971), 87-98.

[6] - Steinitz classes of cyclic extensions of degree $l^{r}$, Proc. Amer. Math. Soc. 49 (1975), 297-304.

[7] L. R. McCulloh, Cyclic extensions without integral bases, ibid. 17 (1966), 11911194 .

Department of Mathematics

College of Charleston

66 George Street

Charleston, South Carolina 29424-0001

U.S.A.

E-mail: carter@math.cofc.edu 